Bull. Austral. Math. Soc.

13A10, 13E10, 13F10, 13м05, 13м10, 16N80,

Vol. 59 (1999) [525-526]

$16 \mathrm{~W} 50,94 \mathrm{~B} 05$

\title{
Artinian rings, finite principal ideal rings and algebraic error-correcting codes
}

\author{
Jilyana Cazaran
}

\section{DESCRIPTION OF THE THESIS}

This thesis contains structure theorems for several special types of Artinian rings, namely the finite rings, commutative Artinian rings with identity, finite commutative rings with identity and semisimple Artinian semigroup-graded rings. Chapter 1 provides an introduction to Artinian rings, semigroup-graded rings and algebraic coding theory. All the theory in Chapters 2 to 5 is new results, some of which have appeared in [1], [2] and [3].

Chapter 2 contains theorems about the generators and weights of certain types of polynomial codes. A class of ideals in polynomial rings is considered which includes all generalized Reed-Muller codes. Necessary and sufficient conditions are obtained for such an ideal to have a single generator. A description is also given of all quotient rings $(\mathbb{Z} / m \mathbb{Z})\left[x_{1}, \ldots, x_{n}\right] / I$ which are commutative principal ideal rings where $I$ is generated by univariate polynomials. Formulas are provided for the minimum Hamming weight of the radical and its powers in the algebra $F\left[x_{1}, \ldots, x_{n}\right] /\left(x_{1}^{a_{1}}\left(1-x_{1}^{b_{1}}\right), \ldots, x_{n}^{a_{n}}\left(1-x_{n}^{b_{n}}\right)\right)$ for an arbitrary field $F$. Most of Chapter 2 appears in [1].

Chapter 3 contains theorems about tensor products and quotient rings of finite commutative rings with identity. Principal ideal rings play a central role in the theory of these finite rings, see [5]. For finite commutative rings $R$ and $S$, necessary conditions are obtained for the tensor product $R \otimes_{\mathbf{Z}} S$ to be a principal ideal ring. These conditions are shown to be sufficient when $R$ and $S$ are principal ideal rings. Conditions are given for the ring $R[x] /(f(x))$ to be a principal ideal ring when $R$ is a principal ideal ring and $f(x)$ is a monic polynomial. For a polynomial ring $Q=R\left[x_{1}, \ldots, x_{n}\right]$, and an ideal $I \subset Q$ generated by univariate polynomials, conditions are obtained for $Q / I$ to be a principal ideal ring when $R$ is a principal ideal ring and $Q / I$ is finite. Conditions are also provided

Received 12th January, 1999

Thesis submitted to the University of Tasmania, July 1998. Degree approved September, 1998. Supervisor: Dr. Andrei Kelarev.

I sincerely thank Professor Rudolph Lidl for inviting me to Tasmania to do the Ph.D. degree and providing financial support in the form of a large Australian Research Council grant.

Copyright Clearance Centre, Inc. Serial-fee code: 0004-9729/99 \$A2.00+0.00. 
for $Q / I$ to be a direct sum of finite fields or Galois rings. Part of Chapter 3 appears in [3].

Chapter 4 contains theorems about radicals of finite rings and principal ideal rings. Radicals are basic structural tools of ring theory, see [4]. For a class $\mathcal{R}$ of finite rings, necessary and sufficient conditions are given for $\mathcal{R}$ to be a radical class and also a semisimple class. Hereditary radical classes are characterized. Conditions are obtained when several such classes consist of principal ideal rings.

Chapter 5 contains structure theorems for Artinian semigroup-graded rings. In [6], Zel'manov proved that if a nonzero semigroup ring $F S$ is right Artinian, then the semigroup $S$ is finite. Chapter 5 has several necessary and sufficient conditions for various $S$-graded rings $R=\bigoplus_{s \in S} R_{s}$ to be semisimple Artinian under certain finiteness conditions on $\operatorname{supp}(R) \subset S$. Various necessary and sufficient conditions are given for $R$ to be semisimple Artinian when $S$ is a semilattice, an inverse semigroup or a Clifford semigroup. All semigroup varieties, $\mathcal{V}$, are described such that the semigroup algebra $F S$ is semisimple Artinian, for every finite semigroup $S \in \mathcal{V}$, where $F$ is an arbitrary field. Most of Chapter 5 appears in [2].

Soft-bounded copies of this thesis are available from the author at the address given below.

\section{REFERENCES}

[1] J. Cazaran and A.V. Kelarev, 'Generators and weights of polynomial codes', Arch. Math. 69 (1997), 479-486.

[2] J. Cazaran and A.V. Kelarev, 'Semisimple Artinian graded rings', Comm. Algebra (to appear).

[3] J. Cazaran and A.V. Kelarev, 'On finite principal ideal rings', Acta Math. Univ. Comenian. (to appear).

[4] B.J. Gardner, Radical theory, Pitman Research Notes in Math. 198 (Wiley, New york, 1989).

[5] B.R. McDonald, Finite rings with identity, Pure and Applied Mathematics 28 (Marcel Dekker Inc., New York, 1974).

[6] E.I. Zel'manov, 'Semigroup algebras with identities', Siberian Math. J. 18 (1977), 787-798.

School of Mathematics and Physics

University of Tasmania, Hobart Campus

GPO Box 252-37

Hobart, Tas 7001

Australia

j.cazaran@utas.edu.au 